\title{
Syringa Josikaea Jacq. und anderes Neue aus der Marmaros.
}

\author{
Von Victor v. Janka.
}

Seit 11. Juli verweile ich wieder in der Marmaros. Um Huszt besuchte ich in Begleitung des Herrn Vágner das sehr ausgedehnte Terrain, welches die - wie von mir bereits im vorigen Jahre gemeldet - für Ungarn neue Viola uliginosa in ungeheurer Masse beherbergt. Von Orobus laevigatus fand ich noch ein paar gerade reife Friichte. - Hernach begab ich mich nach Rahó. Obwohl ich dahin gegen vergangenes Jahr um einen vollen Monat früher kam, fand ich der heurigen Dürre halber die Gentiana Vágneriana im selben überreifen Zustande. Der Einfluss der Dürre beschränkte sich eben nur auf raschere Entwicklung; die sonstige Grösse der Pflanze und der lange über die Corolla herausragende Fruchtstiel blieb sich constant. - Gentiana cancasica M. B. sammelte ich in Masse. Ich orinnere mich, dass Grisebach im "Iter hungaricum" eines ganz besonderen Unterschiedes dieser Gentiana von jedweder G. germanica im Drehungsverhältniss des Blüthenknospensaumes Erwähnung thut. Ich fand unsere $G$. caucasica darin wirklich ganz abweichend von den übrigen mir bekannten Arten. Es decken sich nämlich bei ihr vier Blumenkronzipfel linksseitig mit den Rändern, während der fünfte beiderseits am Rande überdeckt, also auf beiden Seiten den Nachbarzipfeln unterschoben ist. - Carex tristis M. B. traf ich auch massenhaft in den üppigsten gerade in bester Frucht stehenden Exemplaren an. Wenn ich nun annehme, dass dio im Caucasus weitverbreitete $G$. pyrenaica der russischen Floristen, als den Karpathon näher gelegen, mit $G$. Vágneriana identisch ist und noch die Telekia speciosa hinzurechne, die im Cancasus noch grössere Verbreitung hat, wie bei uns, so weist die Marmaros gleich vier cancasisch $\theta$ Pflanzentypen sozusagen auf einem Flecken anf. Noch kann ich das Vorkommen von Plantago montana am Terentin (wo G. Vágneriana gemein ist) mittheilen.

Aus dem oberen Theissthale begab ich mich wieder nach Huszt zurück, mit dom Plane, von da aus nordwärts über Ökörmezó vorzudringen, um dann auf irgend eine Weise Fühlung mit Syringa Josikaea zu suchen, deren nächster Standort ja 8 Meilen nordwestwärts im angrenzenden Beregher Comitate bereits constatirt ist und daher die Wahrscheinlichkeit nahe lag; den Strauch auch in der Marmaros zu entdecken. Die Strecke von Huszt gegen Ökörmezó und darüber hinaus hat noch nie ein Botaniker betreten - selbst unser floissige Vágner nicht, obwohl so viele Jahre hindurch Huszt sein Wohnort war. Man konnte demnach sicher annehmen, ein paar wenigstens für das Comitat neue Funde zu machen. Drei Stunden weit von Husat, schon im Gebirge entdeckte Vágner Geum strictum Ait.; ich fand gleich daranf Potentilla norvegica L. und oin Exemplar oesterr. botan. Zeitschrift. 9 . Heft 1885 . 
eines Bastartes zwischen dieser und Potentilla argentea L. Die übrige Strecke bis Ökörmezö bot ausser überall auftretender Telekia und an einem Orte häufigem Echinops commutatus Juratzka (von mir übrigens auch am Huszter Schlossberg 1884 gesehen) nichts Besonderes, so dass man sich ansonsten in irgend eine entsprechende Gebirgsgegend Oesterreichs oder Böhmens versetzt denken könnte. - Am ganzen Wege versuchte ich auf die mannigfachste Weise Erkundigungen in Bezug auf Syringa Josikaea einzuziehen, aber überall vergeblich. So kamen wir nach Ueberwindung der entsetzlichsten Hindernisse auf einer eben in vollem Bau begriffenen Strasse nach 14 Stunden halbtodt in ökörmezö an. Hier ist ausser bei den schmutzigen polnischen Juden keine Unterkunft zu finden und hätten wir auf offener Strasse übernachten müssen, wenn der hiesige ruthenische Geistliche uns nicht bereitwilligst und auf die gastfreundlichste Weise aufgenommen hätte. Am anderen Morgen (25. Juli) hatte ich das grosse Vergnügen, im emeritirten ehrwürdigen Geistlichen Csopey die hochwerthe Bekanntschaft eines echten UniversalGelehrten zu machen, den ich mindestens bloss mit unserem Nestor Brassai gleichzustellen vermag. Von ihm ward ich auf die erste Spur vom Vorhandensein der Syringa in der Marmaros geleitet. Er sagte mir nänlich, dass er im Dorfe Kelecsény - vier Meilen nordwestlich von hier vor 22 Jahren den damaligen Pfarrer ein noch kaum in Beblätterung befindliches Sträuchlein - in naher Umgebung des Ortes wild wachsend in dessen Garten verpflanzen sah, das or als schon damals ausserordentlicher Blumenfreund und Kenner alsogleich für eine von der gewöhnlichen Syringa verschiedene Art erkannte. Dieser Pfarrer sei seither gestorben, aber der Strauch wäre noch vor 6 Jahren unter dessen Nachifolger vorhanden gewesen und dürfte noch existiren. Alles andere Ausforschen nach dem Bekauntsein der Pflanze unter den ruthenischen Bauern oder nach einer russischen Benennung derselben blieb resultatlos.

Der folgende Tag ward alsogleich für die Fahrt nach Kelecsény festgesetzt -, der laufende aber zu diversen Excursionen, Vormittag (auf die östlichen nahen Bergwiesen) wnd Nachmittag benützt. Bloss die letztere in das am südlichen Ende des Ortes mündende östliche Querthal fiel lohnender aus: hier war Oenanthe banatica Heuff. (übrigens auch bei Huszt, aber von Vággner für Oe. silaifolia genommen), ein Laserpilium, wie $L$. alpinum aussehend, aber der niedrige Standort, wo noch kaum Nadelholz bemerkbar, auffallend, Caltha laeta und eine der Carex pallescens ähnliche, aber kahlblättrige Species etc. etc.

Abends zurückgekehrt erfuhr ich weitere Nachricht über wildes Vorkommen eines der Garten-Syringa ähnlichen Strauches in nächster Umgebung einer Säuerlingsquelle am Wege noch vor Kelecsény, wo die Blüthen dem Ökörmezöer Stuhlrichter im Juni d. J. auffielen. Mit diesen Andeutungen versehen, ward die Entdeckungsfahrt nach Syringa Josikaea während strömenden Regens, der unausgesetzt den ganzen Tag über andanerte, angetreten. Trotzdem ward an 
vielen Punkten Halt gemacht, bald hier, bald dort auf ein paar hundert Schritte seitwärts recognoscirt, aber nirgend etwas bemerkenswertheres beobachtet; die Berge wurden immer niedriger, das Nadelholz weniger. Eine Weile war für mich das baumartige Auftreten von Juniperus communis neu. Es war bereits 1 Uhr vorbei, als wir Kelecsény annähernd, beim ersten Sauerwasser links am Waldrande und hart an der Strasse anlangten, aber am steilen Waldabhange war nicht eine Spur einer Syringa zu erspähen.

Ein paarmal glaubte ich derlei zu sehen; aber gar bald erwies sich's als ein Evonymus oder Rhamnus Frangula. - So verhielt os sich auch bei der zweiten Quelle $1 / 2$ Stunde später. Jetzt blieb nichts mehr äbrig, als im Dorfe selbst nachzuforschen. Da war der Pfarrer nicht wenig erstaunt, als ich nach allerkürzester Vorstellung sogleich, fast ohne mich weiter um ihn zu kümmern, in giessendem Regen seinem Gärtchen zustürmte, vor dessen Eingang ich, sogleich die mir in Ökörmezö explicirte Syringa als S. Josikaea erkennend, in Freudengebeul ausbrach - auf welches hin die ganze vielköpfige (ich glaube auch 8 Mädcben gezählt $\mathrm{zu}$ haben) Gesellschaft ins Nasse gelockt wurde -, mit gezücktem Messer darauf losstürmte und drei grossblättrige Wurzeltriebe abschnitt. Es waren kaum ihrer doppelt so viel; der alte etwa eine Spanne dicke Stamm längst fast vom Boden abgehauen. An einem Seitentriebe sah man an vertrockneten Ueberresten, dass er heuer geblüht habe. Nun erst liess ich mich mit dem Pfarrer in weiteres Gespräch ein. Mein früherer Jubel war sogleich zu Ende, als ich erfuhr, dass der Geistliche vom Vorkommen eines ähnlichen Strauches in der Umgegend absolut nichts wusste und auch verschiedene Bauern, denen die Triebe vorgezeigt wurden, so eine Pflanze in ihrer Heimat gar nicht kennen wollten.

Mittlerweile suchte mich der Geistliche und dessen Gemalin auf das zuvorkommendste zu bewirthon. Ich aber hatte durchaus keine Ruhe, refusirte alle Vorbereitungen zu einem Gastmahle, das die gute Hausfrau improvisiren wollte, gab mich mit einem Glas Schnaps zufrieden und sandte um Herrn Vágner, der mit dem Wagen bei einer jüdischen Schänke der weiteren Dinge harrte. Auch war es schon nahe $3 \mathrm{Uhr}$; es war schon Zeit, bei so schlechtem Wetter auf die Rückkehr zu denken. Da fiel mir ein, dass, als ich gestern bei Tisch den Wunsch äusserte, Marmaroser Diamanten in grösserer Menge zu erhalten, mir der Kelecsényer Notär genannt ward, der solche in Quantität besässe und als sehr gefälliger Mann mich sicherlich damit versehen werde. Trotz der ziemlich weit entlegenen Wohnung des Notärs wollte ich die Gelegenheit nicht unbenützt vorübergehen lassen und mich zu ihm bemühen. - Auch durchzuckte mich noch ein Strahl der Hoffnung, dass vielleicht der Notär Jäger oder Naturliebhaber sei und dass ihm vielleicht eine Syringa in der Gegend aufgefallen sei?! Mit diesen Gedanken trat ich, noch immer die Syringa-Zweige in der Hand, nach 10 Minuten beim Notär Toma Jenö ein, der mit seiner Frau auf gleich liebenswürdige Weise, wie der Geistliche Volosin, mich herzlich bewill- 
kommte und bewirthen wollte, was ich aber bis auf 1 Glas Szilvorium ausschlug, da uns schon früher der Pfarrer gekapert hatte. Nun wurden Notär und Frau auf die Syringa aufmerksam und nach kurzem Wortwechsel war ich des häufigen Vorkommens der Syringa Josikcea in nächster Nähe des Dorfes, also auf Marmaroser Gebiet gewiss. Der Standort ward vom Notär dem mich begleitenden Bauer, der uns auf der Rückfahrt hingeleiten sollte, ruthenisch erläutert und nachdem ich noch eine Schachtel mit Marmaroser Diamanten zu Geschenk erhalten, eilte ich freudestrahlend in die Pfarrei zurück. Nach kurzem Imbiss brachen wir auf, waren nach einer balben Stunde beim vom Ökörmezöer Stublrichter ganz richtig gemeinten Säuerling, von dem an die Thalsohle Moor ausfüllt, in dessen Mitte die gefeierte Syringa zwischen Alnus glutinosa, A. incana, Rhamnus Frangula und Salix aurita, die alle an Individuenzahl viel reichlicher auftreten, zwar häufig aber verborgen vorkommt. Zu dieser Zeit war sie von der Strasse aus auf 100 Schritte wohl nicht erkennbar; zur Blüthezeit indess muss sie jedenfalls von Weitem auffallen.

Um 1/2 6 Uhr waren wir mit dem Sammeln der Syringa, die heuer leider keine Früchte angesetzt hatte, fertig, eine Stunde später erreichten wir das Dorf Ripinye, in gleicher Zeit Szolyma und kamen gegen $9 \mathrm{Uhr}$ Abends bis auf die Haut durchnässt in Ökörmezó an. - Diess die Geschichte vom Aufspüren der Syringa Josikaea in der Marmaros.

Schliesslich habe ich noch eine Entdeckung mitzutheilen, die ich im oberen Nagyágthale oberhalb Also-Bisztra machte: ich fand da an Waldrändern zwischen Sumpfgräsern die nur noch von Simkovics im Biharer Comitate entdeckte Agrimonia pilosa Ledeb. Die ungarische Pflanze stimmt trefflich mit dem, was Körnicke in der Oesterr. bot. Ztschr, 1863 über die Petersburger Exemplare sagt. Es war mir äusserst lieb, dass ich hier in der reichbaltigen Bibliothek des Herrn Vágner darüber gleich nachuuschlagen die Gelegenheit hatte.

Huszt, am 6. August 1885.

\section{Beitrag zur Flora des böhmisch-mährischen und des Glatzer Schneegebirges.}

Von Dr. Ed. Formánek,

k. k. Professor am böhmischen Gymnasium in Brün.

(Fortsetzung).

Senecio barbaraefolius Krock. Saar, Schloss Saar, Neudeck.

- nemorensis L. ampl. Chudobín, Sklené, Ỷákowá hora, Ingrowitz, Neu-Ingrowitz, Jaworek, Heinzendorfer Gründe unterm Schwarz- 